\title{
BRILLIANCE AND CHROMA \\ IN RELATION TO ZONE THEORIES OF VISION* \\ $\mathrm{BY}$ \\ LEONARD Thompson Troland
}

\section{INTRODUCTION}

Ophthalmologists draw a clear line of demarcation between three functions of vision which they call the light sense, the form sense, and the color sense respectively. They find that in visual derangements these three functions may be disturbed more or less independently. However, theorists in the field of physiological optics have not been able to agree that these three functions rest upon separate mechanisms. From some points of view and by certain tests they appear to be distinct, but from other angles they appear to be simply different aspects of an integral process. It is my purpose in the present paper to discuss certain problems relating to the separateness of the mechanisms underlying two of these functions, viz., those of the light sense and the color sense. I shall review and attempt to synthesize certain data previously established by others, but in addition shall present new data of my own bearing upon the problem.

It will be necessary in the beginning to define clearly certain of the terms to be used in the ensuing discussion. A great deal of confusion exists in arguments over visual problems as a result of the absence of a definitely established nomenclature. In the majority of discussions in this field there is a failure to distinguish clearly between psychological, or subjective conceptions, and physical conceptions which relate only to the stimulus. The Colorimetry Committee of the Optical Society in its forthcoming report $^{1}$ has worked out what it deems to be a consistent terminology, and I shall make an endeavor to employ this in the present paper. You have been accustomed to hear the two aspects of visual experience, which I am to consider, described by the terms

* Paper presented to the Rochester Section of the Optical Society of America, October 10, 1921.

1 To appear in a later number of this Journal. 
"brightness" and "color." However, the word "brightness" has a distinct meaning in photometry which should not be confused with that dimension of visual sensation which is defined by the terminal qualities black and white. The preferred term for this dimension, or attribute, of visual sensation is the word "brilliance." A careful consideration of all of the circumstances bearing upon the problem, moreover, seems to make it advisable to employ the word "color" to designate any visual sensation whatsoever, including the neutral, or achromatic, qualities as well as the chromatic ones. In order to distinguish the latter from the former, therefore, we must employ a different word, for which the term "chroma" appears to be appropriate. I may therefore define my problem succinctly as that of the interrelation between the physiological mechanisms underlying brilliance and chroma vision.

\section{Facts Indicating the Independence of Brilliance AND CHROMA}

There is a considerable array of facts which strongly suggest that these mechanisms are distinct from one another. The first consideration which comes to mind is naturally that of the evolution of color vision. Experiments in the field of comparative physiology, such as those carried out by the indefatigable Carl von Hess, ${ }^{2}$ indicate clearly that the invertebrates are totally lacking in chroma vision. Their reactions to radiation are wholly in terms of different degrees in a single dimension which we may suppose to be that of brilliance or apparent brightness. Their vision is achromatic. The consensus of evidence also is that the lower vertebrates, such as fish, are incapable of chromatic discrimination, their differentiated responses to various colors being based wholly upon differences in the brightness effects of the latter. ${ }^{3}$ Many birds and mammals, however, seem to possess the power of true chromatic differentiation between stimuli. The visual capacities of the higher primates are apparently practically

\footnotetext{
${ }^{2}$ See, for example, Hess C. v. Beiträge zur Kenntnis des Lichtsinnes bei Wirbellosen. Arch.f. d. ges. Physiol., 177, 57-109; 1920.

${ }^{3}$ See, for example, Schnurmann, F. Untersuchungen an Elritzen über Farbenwechsel und Lichtsinn der Fische. Zeitsch.f. Biol., 71, 69-98; 1920.
} 
the same as those of man. It appears probable, therefore, that brilliance vision appeared in the course of evolution long before chromatic vision, the latter being superposed upon the former by a process of accretion or of differentiation.

Facts of visual pathology and anomaly also suggest strongly that brilliance vision is more fundamental than chromatic vision. Disease or injury of the nervous mechanisms connected with vision more readily disturbs chromatic discrimination than it does discrimination in terms of brilliance. Congenital partial or total "color blindness" involves a loss or impairment of the capacity for differentiation of chromas without necessarily bringing with it a similar disorder of brilliance judgment. The different types of chromatic blindness can, in fact, be arranged in an evolutionary series ranging from anomalous trichromatism through deuteranopia, or green blindness, and protanopia, or red blindness, to the complete absence of chromatic response which is commonly known as "total color blindness." In these various forms of chromatic blindness it would appear that successively laid down strata of visual mechanism have been destroyed by accidents to the germ plasm, these accidents representing various degrees of atavism. The resulting visual types may be taken to represent actual stages in the evolution of human vision.

However, we do not have to look to pathological or rare cases for evidence that chromatic vision is something superposed upon a more fundamental brilliance vision. In the distribution of the capacity for chromatic discrimination between the center and the periphery of the visual field we seem to discover a replica of the evolutionary process. In the extreme periphery of the visual field chromatic vision appears to be practically in abeyance, all objects, except under conditions of very high illumination, being perceived as neutral in, color. $^{5}$ As the stimulus is moved from the periphery towards the center, chromatic discrimination in terms of blueness 1920.

${ }^{4}$ See Hess, C. v. Die Rotgrünblindheiten. Arch.f. d. ges. Physiol., 185, 147-164,

${ }^{5}$ Ferree and Rand find that red, yellow and blue, but not green, can be perceived as chromatic at the extreme periphery with a sufficient intensity of light. See Ferree, C. E. and Rand, G. The Absolute Limit of Color Sensitivity and the Effect of Intensity of Light on the Apparent Limit. Psychol. Rev., 27, 1-23; 1920. 
or yellowness first becomes possible, this being succeeded at a more central position by an added power of discrimination in terms of redness and greenness. In the center of the field for the normal individual we find complete color vision. It is a very significant fact that in spite of these wide differences which exist between the chromatic perception of various portions of the retina, under conditions of daylight adaptation, the visibility curves representing the brilliance responses of these various portions are practically identical. ${ }^{6}$.

Another very impressive group of facts which indicate the separability of the mechanisms underlying brilliance and chroma is to be found in a considerable number of laws of visual response in which the effects produced by lights of different color are substantially independent of the chromatic aspect and rest almost wholly upon the brilliance factor. One of the most familiar of these laws is to be found in the logarithmic function which connects visual acuity with luminosity. This function, which represents the threshold of the form sense, is seemingly determined, to the first order at least, by the brilliance value of any stimulus independently of its hue or saturation effects. ${ }^{7}$ There are, of course, second order dependencies upon wave-length, as has been demonstrated by Luckiesh, ${ }^{8}$ but these latter dependencies are

${ }^{6}$ See Parsons, J. H. An Introduction to the Study of Color Vision, p. 71, 1915.

7 The proposition that the acuity index depends upon the brightness value of a stimulus, independently of its color, was clearly enunciated by Helmholtz in several places, and had been assumed by previous workers such as Macé de Lapinay and Nicati who employed equality of acuity as a criterion of equality of brightness. Repeated attempts have been made to employ an acuity test as a basis for heterochromatic photometry. König regarded his very systematic work on this subject as clearly substantiating Helmholtz's original conjecture. See König, A. Die Abhangigkeit der Sehscharfe von der Beleutungsintensität, Gesammelte Abhandlungen zur Physiologischen Optik. 1903, p. 391. However, the problem is much complicated by uncertainty in the conditions or method of observation: the proportions of rod and cone vision involved, the exact visibility curves of the observers, and the exact importance of the purely physical chromatic aberration effects within the eye. Dr. Ferree finds a very considerable dependence of acuity upon chroma even when rod vision is excluded. Whether his results, in common with those of Luckiesh, can be explained in terms of the refractive properties of the eye or whether they will require a retinal basis is at present uncertain.

${ }^{8}$ Luckiesh, M. Color and Its Applications, pp. 130-137, 1915. 
directly traceable to the chromatic aberration of the eye which forms a sharper image upon the retina for the mid wave-lengths of the spectrum than for the extreme wave-lengths. Given equally sharp retinal pictures, it would seem that the resolving power of the optical mechanism is determined wholly by the brilliance response without reference to chroma.

Another well known law of this character is that which links critical flicker frequency with the brightness of the stimulus. T. C. Porter found that this frequency, the rate of alternation of a color with black which is required just to eliminate flicker, is strictly proportional to the logarithm of the brightness throughout a range of from 1 to 12800 units of intensity and that the proportionality factor is strictly.independent of wave-length. ${ }^{9}$ Here again, as shown later by Ives, ${ }^{10}$ there are secondary dependencies upon chroma, but these seem to rest upon the fact that there are two kinds of flicker, one a brilliance flicker and the other a chromatic flicker, which are not quite separable at low intensities and rates of alternation. Moreover, the situation is complicated by the different degrees of participation of rod and cone vision in the process as aroused by stimuli of different wave-lengths at low brightnesses, the brilliance response of the rods being much more sluggish than that of the cones. The substantial independence of critical flicker frequency upon chroma is, of course, the basis of the critical frequency method of heterochromatic photometry.

Another function of brilliance which shows very little concomitant dependence upon chroma is the time required for the brilliance sensation to reach its maximum after the first application of the stimulus. McDougall's investigation ${ }^{11}$ indicated that this so-called action time was quite independent of chroma. The much discussed experiments of Broca and Sulzer, ${ }^{12}$ however,

${ }^{9}$ Parsons, J. H., op. cit., p. 96.

${ }^{10}$ Ives, H. E. Studies in the Photometry of Lights of Different Colors. II. Spectral Luminosity Curves by the Method of Critical Flicker Frequency. Phil. Mag., 24, p. 357-362, 1912.

${ }^{11} \mathrm{McD}$ ougall, W. The Variation of the Intensity of Visual Sensation with the Duration of the Stimulus. British Jour. of Psychol, 1, p. 189, 1904

${ }^{12}$ See Nutting, P. G. The Luminous Equivalent of Radiation. Bull. of the Bur. of Stands., 5, p. 293, 1908. 
showed a considerable difference in the rates of rise of sensation due to stimuli of different wave-length compositions. The recent very elaborate measurements of Bills ${ }^{13}$ also show an appreciable difference between colors of the same brilliance but differing hue. However, neither of these investigations, apparently, were made under conditions which insure equal degrees of participation of rod and cone vision for all of the stimuli employed, and the close affiliation which exists between rates of rise and fall, for various stimuli, and flicker frequency, suggests that causes of error in the investigations in question may exist.

A further very important visual function which rests exclusively upon the luminosity of the stimulus is the brightness discrimination threshold. The elaborate measurements made by Königit with a wide range of spectral stimuli demonstrate that for cone vision Weber's constant, and Fechner's law, which is derived from it, are practically independent of chroma. It is probable also that the brilliance contrast effects between color fields of differing brilliance are independent of concomitant chroma or chroma differences. I gather this from qualitative observations of my own, although I have not been able to find any published accurate data on the subject. The investigations of Ives ${ }^{15}$ and others have made it clear that the separate brightnesses of different colors which are mixed additively summate arithmetically without reference to their differences in chroma.

All of the above discussed facts, which indicate that brilliance can act as an independent variable determining other visual functions almost without reference to the accompanying chroma, may perhaps be regarded as somewhat lacking in significance because, to a certain extent at least, they may be considered as definitory of the nature of brilliance. It has been suggested, for example, that brilliance be defined in terms of equal flicker frequencies or in terms of equal acuity results. I do not regard

${ }^{13}$ Bills, M. A. The Lag of Visual Sensation and Its Relation to Wave-Length and Intensity of Light. Psychological Review Monographs, 28, No. 5.

${ }^{14}$ See Nutting, P. G. loc. cit., p. 286.

${ }^{15}$ Ives, H. E. Studies in the Photometry of Lights of Different Colors. IV. The Addition of Luminosity to Different Colors. Phil. Mag. 24, pp. 845-853, 1912. 
this objection as actually capable of substantiation, but, on the other hand, it is significant that most of the laws which we have above considered involve processes of discrimination as essential factors. These discrimination processes undoubtedly depend upon cortical mechanisms which are especially adapted to deal exclusively with brilliance or its underlying physiological correlate, and hence the lack of dependence which the resulting reactions show with respect to chroma might be considered as indicative merely of a highly efficient selective response of these discriminative activities. However, there are other facts to which we can yet appeal which are not subject even to this last objection.

It is a common conviction among students of physiological optics that negative after-image phenomena depend upon retinal rather than upon central changes. These phenomena are usually explained in terms of general or differential retinal fatigue, that is, as results of reduction in the sensitivity of the retinal mechanism to stimuli, this sensitivity being supposedly represented by the concentration of some chemical substance. Negative after-image effects may be divided into brilliance and chromatic aspects. I have personally made a very large number of observations and measurements upon the brilliance aspects of negative after-images produced by spectral or other highly chromatic stimuli. Except under special conditions which I shall discuss in more detail later on I have found these effects to be practically independent of chroma. For example, the duration of negative after images produced by spectral colors and projected upon a reacting field or background of the same spectral color as produced the image are practically the same for all wave-lengths of the stimulus. ${ }^{16}$ It is true that there is a slightly less duration for stimuli lying at the ends of the spectrum than for those lying in the middle, and also that the red after-image has a somewhat longer life than the violet one. However, it seems probable that these secondary differences are due to differences in the sharpness of the primary stimulus images which produce the after-effects, such sharpness

${ }^{16}$ Troland, L. T. Apparent Brightness; Its Conditions and Properties. Trans. of the Illum. Eng. Soc. 5, p. 954; 1916. 
discrepancies being referable to the chromatic aberration of the eye, which influences the distinctness of the retinal picture in the several cases. I have made careful measurements of the time required for brilliance fatigue, or minuthesis, as I have proposed to call it, to reach an equilibrium condition for different spectral stimuli, and find that for colors of equal brilliance this time is practically independent of chroma. Similar statements apply to the degree of reduction of the sensitivity of the visual system which is brought about by this minuthetic process in any specified time or at equilibrium with stimuli of equal brightness. The general laws of brilliance minuthesis, in other words, are substantially independent of chroma. I shall return to a consideration of such further special laws later on.

\section{The Problem in the Light of Classical Theories}

Having reviewed the above facts bearing upon our problem, let us now turn to a discussion of the most important theoretical treatments dealing with the interrelation of brilliance and chroma vision. The two salient theories of vision, those of Hering and of Young and Helmholtz, ${ }^{17}$ involve radically distinct conceptions of the relation holding between brilliance and chroma. Hering, in the original formulation of his theory, regarded brilliance as identical with whiteness and, therefore, as proportional to the degree of excitation of the white process, in his theory, or of lack of excitation of the black process. Later on, however, he introduced the conception of the specific brightness of colors according to which the red and yellow processes of his theory possess a brilliance-producing power while the green and blue processes have a negative capacity in this respect, or are "specifically dark." The total brilliance effect according to Hering's view, therefore, represents the algebraic sum of contributions made by all six processes, the white, red, and yellow adding to, while the black, blue, and green subtract from the total. Even in accordance with this specific brightness theory, however, the preponderant contribution appears to be made by the black-white process,

${ }^{17}$ For expositions of these two theories see Parsons, J. H., op. cit., Part III. 
so that Hering's hypothesis seems consistent with the existence of a very considerable independence of brilliance function with respect to chroma. A residual dependence, however, should be expected, and this should be of the order of magnitude of the difference between cone and rod vision luminosity distribution over the spectrum, or between the photopic and scotopic visibility. curves. It was in order to explain these differences that Hering originally introduced the specific brightness theory.

The Young-Helmholtz theory, being characterized in general by a less subtle psychological analysis, than that which governed Hering's conjectures, makes assumptions concerning the interrelations of the brightness and chroma mechanisms which are far simpler and more naive. Helmholtz himself and also his followers, König and Dieterici, paid relatively little attention to the relations of photometric measures to color-mixture data. The latter two investigators, for example, although their determinations of the three color sensation curves are probably the most thorough on record, provide us with no measures whatsoever of the relative photometric values of the unit in which their sensation curves are expressed. This criticism, however, does not apply to Abney, ${ }^{18}$ whose recent death robs us of one of the most painstaking investigators of visual phenomena in the light of the Helmholtz Theory. This theory, as is well known, makes the chromatic aspects of color vision depend upon the proportions of excitation of three elementary mechanisms. It is very natural to hold that the brilliance accompanying any complex or simple excitation is simply the sum of the excitation values of the components which are involved.

The treatment of brilliance or luminosity value as the sum of the color excitation values is not only a theoretically obvious hypothesis, but is a straightforward development of the data involved in the case; although in spite of this fact these data cannot be regarded as proving the physiological identity of the brilliance and chroma mechanisms. When a spectrum having a characteristic luminosity distribution,- - for example one possessing

${ }^{18}$ See Abney, W. de W. Researches in Color Vision and the Trichromatic Theory, 1913. 
equal energy values, for all wave-lengths, in which case the luminosity distribution would be proportional to the visibility curve,-is matched by varying mixtures of three elementary stimuli it is of course a necessary consequence of the additive property of luminosities that the sum of the luminosity distributions of the three elementaries should yield the original luminosity distribution of the spectrum which was matched. However, this same result should be expected if the luminosity values of the elementaries do not represent integral aspects of the color excitation processes, but simply more or less accidental associates of these excitation values the magnitudes of which are determined by the exact point in the spectrum from which the three elementary stimuli are picked. The brilliance process necessarily has a characteristic distribution over the spectrum, represented approximately at least by the visibility curve. Similarly, the three chromatic processes also have their own characteristic distributions, and a stimulus taken at any point in the spectrum will naturally pick up the chromatic and the brilliance activities in a fixed ratio, and this ratio will enter as a constant in all subsequent color-mixture operations. These experimental facts, therefore, provide us with no basis for distinguishing between the inherent brilliance assumption and the idea that brilliance depends upon a mechanism distinct from that governing chroma.

It does seem to me, however, that the relative magnitudes of the three coefficients which are empirically found to represent the above mentioned proportionality between the brilliance and chromatic powers of any stimulus, do have some bearing upon the probability of the inherent brilliance assumption. When the elementaries to be mixed are red, green and blue, practically all of the luminosity of the spectrum appears to depend upon the red and green, the blue contributing only about one per cent. of the total. Depending upon the exact elementaries which are selected, either the red or the green may greatly preponderate over the other. Such large discrepancies between the chromatic and the brilliance powers of the elementaries would suggest that the underlying mechanisms of the two functions are actually distinct, these coefficients representing an arbitrary association of the two. 
Another consideration which points in the direction of an independence between the brilliance and chromatic mechanisms is to be found in the very perfect symmetry of the retinal visibility curve. ${ }^{19}$ This latter curve is obtained by correcting the ordinary normal visibility curve for the selective absorption of the ocular media, and should be regarded as representing the spectral sensitivity of the brilliance process by itself. It is improbable that independent spectral distributions having maxima in different portions of the spectrum, such as those of the three fundamental chromatic excitations, should be capable of summating to yield the very perfect, symmetrical curve which represents the retinal visibility function. It is true that the empirically obtained chromatic excitation curves do actually summate in this manner, but it seems probable that their exact form is dictated by the relations between a symmetrical brilliance function and chromatic response curves which in their true physiological forms do not actually summate to yield a symmetrical curve.

\section{Studies on the "Abney Effect"}

Although a direct analysis of the color-mixture system does not permit us to differentiate between the two hypotheses which we have under consideration, it is far from being impossible to find a means of testing between them. It would seem likely, a priori, that there must be some way by which the casual association of a chromatic and a brilliance process, such as that which a theory of the Hering type supposes to exist for a stimulus of any given wave-length, could be broken down. A method of actually dissolving this association presents itself in experiments of brilliance fatigue with lights of different color. Exposure of the retina to continued stimulation by radiation of any wavelength composition brings about a radical reduction in both the chromatic and the brilliance responses of the eye. In the course of the exposure the apparent brightness of the color is reduced by an asymptotic process to a level which is lower the higher the

${ }^{19}$ See Troland, L. T. loc. cit., p. 955-957. 
intensity of the stimulus and which at moderately low intensities may amount to the elimination of ninety per cent. of the original value. At the same time the color, if it be chromatic, changes in saturation and usually also in hue. A careful study of the laws governing these simultaneous brilliance and chromatic fatigue processes, or as I have proposed to call them, minutheses, ought to throw a clear light upon the question as to the fundamental dependence or independence of the two.

I am not acquainted with any exact data bearing on the question as to the identity of the laws of minuthesis for brilliance and chroma. Qualitative observations of my own indicate that the two processes do not occur at the same rate or exhibit the same constants: I hope in the near future to make some careful quantitative measurements on the simultaneous courses of these two processes. It ought to be particularly instructive to carry the minuthesis to its asymptotic limit both for the brilliance and chromatic aspects of the sensation, and then to observe the course of recovery of these two attributes. Qualitative observations indicate that they do not recover at the same rate.

One very obvious method, resting upon the facts of minuthesis, of attacking our problem is as follows. Suppose that we fatigue the retina by a spectral red of given intensity and that we measure the diminution in apparent brightness brought about by this minuthetic process. Let us then throw upon this fatigued area a spectral green stimulus and again measure the reduction in apparent brightness which has been produced for this second stimulus. Since the spectral red should be expected to fatigue the elementary red sensation mechanism much more than the elementary green mechanism we should anticipate on the AbneyHelmholtz assumption that the luminosity reduction for the green stimulus would be much less than for the red one. On the other hand, if brilliance and chroma depend upon distinct mechanisms the factors involved, so far as brilliance is concerned, should be the same in the case of the green as in that of the red, and consequently the percentage reduction of the two should be identical. Another way of stating the proposition in a very general form is to say that fatigue with colored stimuli should be 
expected on the Abney-Helmholtz hypothesis to modify the form of the visibility curve, whereas on the alternative theory this should not occur.

Abney himself carried out experiments of this general character, and his results indicate clearly that a modification in the form of the visibility curve actually does occur. ${ }^{20}$ Moreover, according to Abney's analysis, the change in question is exactly such as would be expected in accordance with his own hypothesis. His results, so far as they go, are clearly in the direction of substantiating the idea that brilliance and chroma depend upon the same mechanism. However, Abney's conditions of experimentation were such as still to leave some doubt in our minds as to the exact significance of his results. In the first place, the size of field which he employed was evidently such as to permit a considerable amount of rod vision to be involved. Different degrees of participation of rod and cone vision accompanying the application of various spectral stimuli yield results very similar to those obtained in Abney's experiments. The effects obtained by Abney were apparently small, although the smoothness of his curves indicates a precision which is astonishing for heterochromatic comparisons of the type involved in such investigations. Moreover, the contrasts in color saturation which appear in such experiments may readily be confused with brilliance contrasts, provided the latter are small. However, the most serious objection to Abney's work lies in the fact that he neglected, in his experiments, to determine the absolute degree of fatigue, his data giving simply the proportionality between the two compared colors. Although his results may indicate some degree of interdependence of brilliance and chroma, we cannot from his data determine whether the relation is of the exact magnitude which is required by his own theory or not.

I have particularly been led to doubt the significance of Abney's results because of the outcome of certain experiments of my own. Several years ago at Nela Park I carried through a series of minuthesis measurements ${ }^{21}$ with spectral colors the conclusions

${ }^{20}$ Abney, W. de W. op. cit., pp. 371-380.

${ }^{21}$ As yet unpublished. 
of which were that the brilliance minuthesis brought about by one spectral color carried over without change to any other such color, or in other words, that the minuthetic effects of different spectral colors of the same brightness are identical as tested by all colors. Large saturation contrasts existing in my experiment, however, would have made impossible the reliable detection of apparent brightness contrasts much less than twenty per cent in magnitude, although I employed the constant error technique and was able to compute statistical differences less than the threshold. The upshot of my measurements was that if any Abney effect,- - as we may call it,- - existed, it was smaller than the photometric threshold in my comparisons. More recently Mr. C. H. Langford and I have taken up this problem anew in the Harvard Psychological Laboratory. Mr. Langford has made preliminary observations of a qualitative nature employing a considerable number of subjects to determine whether or not the Abney effect is observed. He finds that the majority of persons experience such an effect, although it is so small that the observer of ten has difficulty in determining whether the contrast is one of brilliance or of saturation. At the same time there are some persons who report a reversed Abney effect. I have observed the latter myself quite strongly with certain combinations of stimuli. This reversed effect, so far as it goes, would point in the direction of the Hering theory of specific brightnesses combined with the doctrine of antagonistic colors. It is quite probable, however, that the reverse phenomenon is attributable to a failure exactly to balance photometrically the two stimuli of different color which are utilized in the fatigue phase of the experiment.

Since one of the principal difficulties which is encountered in experiments of the type just described consists in the existence of a very large saturation contrast superposed upon the brightness contrast which it is desired to study, it occurred to me that a flicker method might be applied to the problem, the saturation contrast being eliminated by fusion in accordance with the well known principle of the flicker photometer. Mr. Langford and I have carried through a systematic series of observations employing this method, the two color stimuli which were used being an 
extreme spectral red or its equivalent, and a minus red obtained by use of the Wratten No. 44 filter. The procedure was as follows. The retina of one eye was first fatigued for three minutes to a two degree field of the red, fixation being kept constant on the center of the field. The red stimulus was now alternated with the minus red, or green, in the same field, and the intensity of one or the other of the two stimuli was varied until the minimum or zero flicker point was found. The intensity of the variable stimulus required for this flicker match was recorded. At another time a similar flicker match was established in the absence of minuthesis by the red stimulus. Two photometric values were thus obtained, the one standing for the brilliance of the red relative to the green with minuthesis, and the other for the same relationship substantially without minuthesis. In parallel with these two measurements, determinations were made of the degree of minuthesis which resulted from the three minute fatigue exposure, this being accomplished by fatiguing a semicircular field and matching this in brilliance by variations in the intensity of a stimulus projected upon the adjacent semicircle at the termination of the fatigue exposure.

From the data thus obtained, using Abney's color sensation curves and the known spectral distributions of the stimuli which were employed, it was possible to compute not only the intensity of the Abney effect which actually appeared to two observers, but also that which should be expected theoretically in accordance with the Young-Helmholtz assumptions. This latter value is approximately 75 per cent., representing the depression of the apparent brightness of the red relative to that of the green. The values actually found, however, were for one observer only 3 per cent. and for the other 5 per cent. The empirical results, therefore, are in radical disagreement with the Young-Helmholtz assumptions as interpreted by Abney. They indicate that the linkage between brilliance and chroma is far less thorough-going than is supposed by these latter theoretical interpretations. Indeed the Abney effect is so small as to suggest that there is actually no affiliation whatsoever between the two functions, on the supposition that the values for the effect actually found are simply due to errors of 
observation. However, considerable care was taken in the experiments to eliminate all asymmetries in the technique, and computation of the probable error of the average results indicates that the magnitudes actually found probably have some significance. The results would seem more consistent with an hypothesis of Hering's type in which the main body of brilliance sensations is attributed to a single process independent of the chromatic excitations although there is a slight residual contribution of brilliance due to the latter. It is possible, however, to reconcile the results with the Young-Helmholtz Theory if we suppose that the three chromatic spectral distribution curves overlap in the spectrum much more extensively than has been assumed by Abney and other interpreters of the Young-Helmholtz Theory. This overlap, however, in order to explain our results, would necessarily be so great that all three of the chromatic curves would differ only slightly from the visibility curve. There are other data which indicate that the actual overlap is greater than assumed by Abney, and on this hypothesis our data could be employed to compute the magnitude of this overlap.

\section{The Dimming Effect and the Association of Brilliance WiTh CHROMA}

In the foregoing discussion $I$ have supported the thesis that the mechanisms underlying brilliance and chromatic vision are distinct, and have attempted to refute the doctrine of the YoungHelmholtz Theory which identifies these mechanisms. I wish to turn now to certain phenomena which, $I$ believe, are quite new and which point in the opposite direction, indicating a very strong affiliation between the brilliance and chromatic functions. These phenomena appear under the influence of sudden changes in the brightness of the stimulus field. The fundamental processes which are involved are probably identical with those of effects which I have previously described elsewhere in conjunction with such brightness changes. ${ }^{22}$

22 Troland, L. T. Preliminary Note; The Influence of Changes of Illumination upon After-Images. Amer. Jourr. of Psychol., 28, pp. 497-503; 1917. 
The conditions for the observation of the phenomena in question, in relation to our present problem, may be described as follows. :The retina is first fatigued by exposure to a bipartite field consisting of two semicircular areas of different color, for example a red and a green, of equal brilliance. Fixation is constantly directed to the center of the dividing line of this field. At the end of the fatigue phase of the experiment the entire circular field is converted into a single color, ordinarily that of one of the fatigue phase stimuli. This is an ordinary procedure for the study of the Abney effect, and this effect, if it manifests itself at all, should be visible on the homogeneous field at the moment of the removal of the additional color which was present in the first phase. In my own experience, as previously stated, however, practically no brilliance difference exists between the two halves of the field. The Abney effect, if it appears, will be of the order of magnitude of five per cent. If now the stimulus field be suddenly darkened or dimmed in intensity, say to about one quarter of its initial value, a very strong brilliance contrast will often appear. The degree of this contrast depends upon that of the dimming as well as upon the absolute brightnesses of the stimuli, and it is furthermore dependent in a very important way upon the exact color pairs which were employed in producing the original minuthesis. For some color pairs the contrast is practically absent, but for others it is very high, amounting often, I should estimate, to a difference of ninety per cent. In other words this dimming technique, or test, brings out an Abney effect, or analogous phenomenon, which is of the magnitude which should actually be expected from theory for the case of stimuli not varied in intensity. These results seem to indicate inevitably that there is a fundamental linkage between the brilliance and chromatic mechanisms, which linkage manifests itself, however, only under these special conditions.

I have made some preliminary experiments on the influence of various combinations of spectral colors upon the effect in question. Four spectral colors, representing red, yellow, green and blue, were selected and each of these were made up into a pair with each other and in successive series of observations the minuthetic effects of all of these pairs were projected upon each of the 
four spectral stimuli as reacting excitations. The results form a rather complex system but in practically every case in which a red was involved a strong brightness contrast appeared during the dim phase of the experiment. The indications, therefore, are that the red chromatic excitation is affiliated with the brilliance process in a unique manner. ${ }^{23}$ The brilliance contrasts in the case of the red in comparison with other stimuli were in the direction coinciding with an expected Abney effect. However, the brilliance contrasts appearing in the case of the blue stimulus in comparison with certain others were in an opposite direction.

It is not my purpose in the present paper to attempt a thorough explanation of these complicated relationships. It will be necessary to accumulate a considerable mass of quantitative data before this can be accomplished with any degree of satisfaction. However, it is necessary as a portion of the argument here to consider briefly a certain general hypothesis concerning the mechanism underlying these dimming effects. I have previously studied experimentally in great detail similar phenomena which appear upon dimming a homogeneous circular stimulus field upon one half of which is projected a negative after-image produced by the same stimulus but of lesser area. Under such conditions a a very strong initial brilliance contrast exists between the two halves of the field, since one half has been fatigued to brilliance and the other has suffered no minuthesis whatsoever. In the course of a minute or so, depending upon the length of the fatigue exposure, this brilliance contrast disappears, owing to the reduction of the fresh area to a level of sensitivity substantially similar to that of the other half of the field. If now, however, the field be dimmed, the brilliance contrast returns with great vividness. On maintaining the field at the dimmed intensity, this brilliance contrast rapidly disappears. If, next, the intensity be restored to its original value a brilliance contrast reappears, but this time in the opposite direction from that which characterized the original effect and also that obtained by dimming. In other

${ }^{23}$ In the Young-Helmholtz Theory, as interpreted by the majority of its exponents, this uniqueness of the red would probably consist in its being the only chroriatic process which is capable of being excited in isolation from others. 
words there is a reversal of the brilliance contrast during brightening of the field. If the bright state is maintained, the brilliance contrast again fades out. This process of dimming and brightening with rejuvenation of the brilliance contrast can be carried out over and over again.

On account of the fact that these brilliance contrasts which result from changes in the brightness of the stimuli are ephemeral in character the most reasonable conception of their nature would regard them as dependent upon differences between the rates of fall and rise of the excitations in the two halves of the field. Upon or during dimming the brilliance in the more minuthesized half of the field drops more rapidly than in the less minuthesized half, while upon or during brightening the rise is more rapid in the former than in the latter. This principle can be stated in terms of resistance of the excitations in the two halves of the field to change in their magnitudes. The resistance to change, whether in decrease or increase, is apparently less in the more minuthesized field than in the less minuthesized one. Given sufficient time, both sides of the field reach the same asymptotic limit either in the process of increase or of decrease, but during the course of their change a difference appears between them due to the greater speed of change of one as compared with the other.

If we turn now from an abstract consideration of the experimental results to the neural mechanism which is responsible for them we find that the most plausible portion of this mechanism in which to look for the resistance changes above considered consist in the so-called synapses, or nerve junctions, which enter into the conductional processes of the optic nerve and tract. Physiologists are accustomed to regard there synapses as seats of a variable resistance or conductance. In general, exercise, or the passage of a nerve current, through the synapses, reduces their resistance. This principle is obviously in harmony with the relationship of our dimming and brightening phenomena, since it is always the more fatigued, or more exercised, portion of the retinal field which exhibits the greater facility of change; which, in other words, darkens or brightens the faster with the corresponding changes in the stimulus intensity. We are therefore led to suppose that the 
mechanisms underlying these dimming and brightening phenomena are localized in the nerve synapses rather than in the retinal receptors.

\section{Explanation in Terms of a Zone Theory of Vision}

In the considerations of the last paragraph we find an indication of the manner in which we may hope to resove the paradox established in the present paper. This paradox is the outcome of two sets of data, one of which seems to indicate the complete, or at least the very approximate, independence of brilliance function with respect to chromatic function. The other system of data, centering around effects resulting from intensity changes in the stimulus, point in exactly the opposite direction, necessitating the supposition that the brilliance and chromatic mechanisms are very closely affiliated. Even a cursory examination of the mechanism underlying visual sensation and perception reveals its extreme intricacy. The prevailing theories of visual processes err in many respects, but fundamentally in their tacit assumption that the visual mechanism is simple, or can be so regarded. The first step in the analytic description of the complex mechanism which we find in the eye and its nervous appendages would appear to be to divide up the propagational or conductional system in which it consists into successive stages or zones. The first of these may be considered to be the object in space before the eye, the second the radiation which is sent off from the object to impinge upon the cornea, the third stage would consist in the refractive adventures of the radiation in the ocular media, the fourth the photochemical changes occurring in the retinal receptors, and the fifth the reaction of the photochemical end products with the optic nerve fibers to initiate the visual impulses. Following thereafter comes a series of nerve conduction stages or zones involving successive synaptic and nerve fibre activities leading finally through the subordinate ocular motor nuclei of the corpora quadrigemina and other lower visual centers, to the visual projection areas of the cerebral cortex. Finally there are the complicated connections of the visual projection areas with various association areas of the cortex. It is probably only in conjunction with processes 
occurring in these latter association areas that chroma and brilliance as aspects of visual consciousness are aroused.

It is clear that visual effects observed in consciousness may depend upon any one or any combination of the mechanisms lying in these successive zones of the visual conduction apparatus. One criterion, or test, such as flicker, may bring out the peculiarities of the mechanism in a certain zone such as, for example, the cortex, whereas another test, such as our dimming procedure, may emphasize the characteristics of some other zonal mechanism, say for example the mechanism of certain synaptic regions which lie afferent to the cortex. Other phenomena, such as the three color sensation curves, may rest principally upon the characteristics of retinal mechanisms. By a proper selection of tests or procedures we might hope to be able to isolate the characteristics and internal relationships of any one of these visual zones. The resolution of our paradox concerning the interrelations of brilliance and chroma would therefore seem to lie in the suggestion that the retinal mechanisms underlying brilliance and chroma respectively are nearly or quite independent of one another, but that the synaptic or certain nerve conduction mechanisms which are subsequent to the retinal processes involve a very definite linkage of the factors which transmit the values of these distinct retinal excitations to the cerebrum.

This doctrine of the existence of zones in the visual mechanism is by no means a new one. It has been recognized by practically all visual theorists, although very few of them have made any use of it since they have tended to suppose that, although the mechanisms involved in the separate zones were separate, they were nevertheless quite similar in character to one another and connected by a point to point correspondence. The theory of Donders, ${ }^{24}$ however, is a definite exception to this rule. Von Kries has also advocated a zone theory which makes the retinal apparatus different in character from that of the cerebrum. The most serious of all theories of this type which has yet appeared, however, is that of

${ }^{24}$ Cf. Parsons, J. H., op. cit., p. 270. 
Schjelderup, ${ }^{25}$ a very recent, and apparently a very important, addition to our vast collection of visual speculations. Schjelderup's theory is worthy of careful study by all students of the visual mechanism.

Schjelderup divides the visual apparatus into three successive zones; those of the retina, the cerebral cortex, and an intermediate stage (Zwischenprozesse). He recognizes the correctness of Hering's analysis of the visual qualities into the six psychological primaries, white, black, red, yellow, green, and blue. In the cerebral zones there are, according to his view, six distinct activities which are in one to one correspondence with these six psychological primaries. There is no element of linkage or identity between these six cerebral mechanisms in and for themselves. In the intermediate zone, however, the activities which correspond with the six psychological primaries, although still in a one-to-one relation with the latter via the cortical elements, are arranged in antagonistic pairs. The black- and white-representing activities are linked together and one of them cannot be affected without influencing the other. The same consideration holds true for the pairs red and green, as well as for blue and yellow. In cases of color blindness due to the dropping out of any one of these intermediate zone processes the antagonistic activities must disappear together. However, in the case of color blindness due to cortical derangement an independent dropping out of elementaries corresponding to antagonists in the intermediate zones is possible. In the third or retinal zone three separate mechanisms are supposed to exist, each having a characteristic response curve to different wave-lengths in the spectrum. One of the retinal mechanisms responds by a process of oxidation to all of the wavelengths of the spectrum but to a varying degree which is represented approximately by the visibility curve. The activities of this mechanism are transmitted exclusively to the white-representing process of the intermediate zone. A second retinal mechanism responds by an oxidative process to the long waves of the spectrum but by an opposed or reductive process to shorter

${ }^{26}$ Schjelderup, H. K. Zur Theorie der Farbenempfindungen. Zeits. für Sinnesphysiol. 51, 19-45; 1920. 
waves, ending approximately at the blue of the spectrum. The oxidative phase of this mechanism transmits its energy not merely to the red-representing process of the intermediate zone but also to a certain extent to the white- and yellow-representing factors of the latter zone. The reductive phase of the same retinal activity also transmits its response not merely to the green-representing element of the intermediate zone but also to a certain extent to the black and blue components of the latter. A third retinal mechanism also shows opposed oxidative and reductive reactions to different wave-lengths with maxima for these respective phases of its activity localized approximately at the yellow and blue, the energies of the oxidative response being transmitted to the green and yellow factors in the intermediate zone activity while the reductive response influences the blue and red components of the intermediate stage. The relation between the retinal zone and the intermediate zone is obviously not a plain one-to-one correspondence but a far more complicated arrangement.

By means of the mechanism thus sketched, which in my opinion is by no means too complicated to represent the actual system of visual response, Schjelderup is able to explain all known forms of color blindness, including not only the common types which are considered by the Hering and Young-Helmholtz Theories but also the rarer forms for which these latter theories are powerless to account. The general nature of the interrelation which Schjelderup's hypothesis postulates as existing between the chromatic and brilliance producing mechanisms promises to be of assistance in the attempt to explain many of the facts which we have considered in the present paper. The retinal mechanism which is associated most closely with the red chromatic process is also linked with the brilliance producing activity, a fact which suggests a possible rationale of the brilliance contrasts which are brought out by the dimming procedure whenever a red enters into a comparison with other colors. The association of the retinal mechanism which is most intimately connected with the green chromatic process with the black producing excitation also suggests a possible explanation of the reversed brilliance contrast which appears in the dimming experiment when blue is contrasted with green or 
yellow. However, it is not my purpose in the present paper to attempt a detailed development of these possibilities.

The moral of the foregoing discussion is that to arrive at a thorough understanding of the mechanism or theory of visual response, it is absolutely necessary to take into consideration the zonal structure of the mechanism which is involved. The three zones of Schjelderup's hypothesis are not too many to account for the actual maze of facts which we encounter. Anatomical analysis shows that many more than three are inevitably concerned in the total process. All of the many visual theories which have been propounded probably have some truth in reference to some zone of the visual process, and it is possible that a sufficient number of zones actually exist to permit each of these multudinous theories to be substantially true for at least one of the zones, leaving other zones open for its opponents.

HARVARD UNIVERSITY

Cambridge, Mass. 